\title{
CORSIKA below the knee
}

\section{Tadeusz Wibig*}

Faculty of Physics and Applied Informatics, University of Lodz, Pomorska 149/153, 90-236 Łódź, Poland.

E-mail: tadeusz.wibigafis.uni.lodz.pl

Extensive Air Showers (EAS) induced by cosmic ray particles of very low energies due to the very steep cosmic ray energy spectrum dominate the secondary particle flux measured by single detectors and small shower arrays. Such arrays connected in extended networks can be used to search for potentially interesting spatial correlations between showers, which may shed new light on the nature of ultra high-energy cosmic rays. Quantitative interpretation of showers recorded by small local arrays requires a different methodology than that used by ordinary big EAS arrays operating in the 'knee' region and above. We present 'small EAS generator', semi-analytical method for integrating cosmic ray spectra over energies of interest and summing over mass spectra of primary nuclei in arbitrary detector configurations. Results on the EAS electron and muon fluxes and particle density spectra are given.

$37^{\text {th }}$ International Cosmic Ray Conference (ICRC 2021)

July 12th-23rd, 2021

Online - Berlin, Germany

\footnotetext{
*Presenter
} 
The idea of small autonomous Extensive Air Shower (EAS) experiments was first explicitly presented by Linsley in 1885 [1] in the context of synchronization of large detector arrays covering tens and hundreds of square kilometers to record showers of the highest energies whose fluxes are so small that only giant instruments can produce meaningful results in a reasonable time. It has been realized to some extent practically in triggering systems of the largest EAS instruments such as the Pierre Auger Observatory or Telescope Array. The complex linkage of local triggers allowed to measure practically the UHECR fluxes of one particle per square $\mathrm{km}$ per century.

Building further similar facilities for higher and higher energy cosmic ray measurements is practically unjustified today. For the measurements of above Greisen-Zatsepin-Kuzmin cut-off new techniques are necessary, such as satellite or radio measurements, which are being worked on by dozens or hundreds of scientists from many countries. On the other hand, recently there has been an increased interest in small-scale EAS experiments designed to satisfy young people's scientific curiosity and develop their interest in science. Small local (school) EAS arrays can play an important role in education. They are one of the very few possibilities of tangible "hands-on" type of learning about nuclear physics, modern high-energy physics, and physics of elementary particles for high school students for whom such knowledge is generally given only indirectly, thus creating its image as something very distant, mysterious and unattainable for the average mortal, as a kind of secret knowledge, modern magic. This leads to the perception of science and physics in particular as something potentially dangerous, which it would not hurt to oppose when the opportunity appears. Resistance and protests against nuclear power plants are an obvious manifestation of this.

In many countries attempts are being made to introduce such projects in schools to stimulate interest in cosmic ray physics. In some this has already been done on a smaller or larger scale, to name some: HiSPARC [2] in the Netherland, WALTA, [3], NALTA, ALTA, [4], SALTA, CZELTA, [5], SKALTA, CHICOS, [6], CROP, CosMO [7] or Maze [8, 9]. From a technical point of view, such small arrays are not a serious problem. A few typical scintillation detectors connected to a simple triggering, monitoring and recording device and the problem is solved. However, since the idea of such projects is to popularize such arrays, and preferably to combine them into one big system, the cost of appropriate equipment for one school starts to play a significant role.

The mere fact of the simultaneous appearance of some signals in several detectors is not fascinating, although it may be interesting to some. It starts to be really interesting from such a fact, or rather from a set of such facts, from a registration that lasts continuously for days, weeks, or even months or years, finally something starts to emerge which is actually knowledge about the surrounding reality. To go from direct measurement to its interpretation, appropriate tools are needed and such tools should be provided to students and teachers together with clear and comprehensible documentation and user instructions.

The minimum number of detectors in one station is of course 2 , as it was in the famous experiment of Auger and Maze in $1938[10,11]$ and as it is now in the stations of the HiSPARC project [2]. For stable operation and full control over background and random coincidences, however, the number of 3 detectors seems to be more secure. However, to try to say something about the size of the shower, we should have more detectors to study the frequency of double, triple and higher coincidence events. By optimizing performance and price we believe that stations with 4 detectors seem to be the best.

Small showers surely pose fundamental problems of interpretation. It is of course impossible to 
localize the shower axes. Besides, with such a small number of detectors ( 3 or 4 ) localization even at higher particle densities on detector sites (which could be the case of larger showers) would be questionable anyway. For 3-4 detectors set up in small distances (about $10 \mathrm{~m}$ ) it is also unrealistic to determine the directions of arrival of showers. In the absence of direction and axis position, event-by-event analysis does not give any meaningful results. What we can sensibly measure is the frequency of observations of various coincidences. The measurement times will allow us to study how the frequency of registration changes with time, which can be a basis for various studies conducted by groups of students, such as variability as a function of atmospheric parameters, day/night variability, and dependence of various "everyday life" quantities on the observed cosmic ray flux. As far as we know, no one has yet investigated the dependence of the COVID-19 infection rates on the cosmic ray flux.

From a fundamental physics point of view, it might be very interesting to combine many (the more, the better) instruments into one network and search for the existence of correlations in real time. Observation of pairs of large showers at distant locations could indicate via the Gerasimova-Zatsepin effect $[12,13]$ existence in the cosmic flux at the highest energies of heavy nuclei. The cosmic ray mass composition in this area still remains a mystery waiting to be solved. The observation of other correlations could suggest the existence of more exotic objects, such as the Cosmic Ray Ensembles sought by the CREDO Collaboration.

Analysis of data from small arrays requires specific methods. Methodological limitations do not allow to use typical for cosmic ray experiment methods of processing data. The interpretation of EAS registrations, in any case, is based on computer simulations.

An obvious element necessary to simulate the passage of shower through the atmosphere is a model describing the high energy particle collision and the secondary particle production. The modeling of strong interactions has a long history. All models on the market today were developed a long time ago and are constantly being refined and improved with the release of new data. The model needs to be implemented into the geometrical structure of the transport through the Earth's atmosphere. Complementing them with a much better known formalism of electromagnetic cascades and descriptions of other more and less important processes leads to processes simulating the development of EAS. Today, one of the most widely used programs for this purpose is the CORSIKA program[14, 15] developed over 30 years ago in Karlsruhe for the KASCADE experiment[16, 17]. Since then this program has been significantly extended and developed and is now used also for simulations at the highest observed energies (even up to $10^{21}$ $\mathrm{eV})$. But small local arrays need to be simulated with energies at the other end of the very steep cosmic ray energy spectrum. The low-energy end of the spectrum is truncated around energies of a few $\mathrm{GeV} / \mathrm{n}$, which is primarily due to solar modulation. Of course, atomic nuclei with kinetic energies of $1 \mathrm{GeV}$ are not capable of causing what we might call a Extensive Air Shower, or even a shower at all. The products of the interactions initiated by such particles would have no realistic chance of reaching the earth's surface.

CORSIKA allows one to do so. There is no obstacle to try to determine the chance to observe a charged particle remaining from a proton initiated cascade starting with the energy of, say, 10 $\mathrm{GeV}$. The chance of seeing anything is surely very small, but we should recall that the flux of such particles is large indeed.

The first and undoubtedly most important parameter of a shower is its size which the CORSIKA 
program provides. We can define the size separately as electron and muon size as the number of electrons or muons at the observation level.

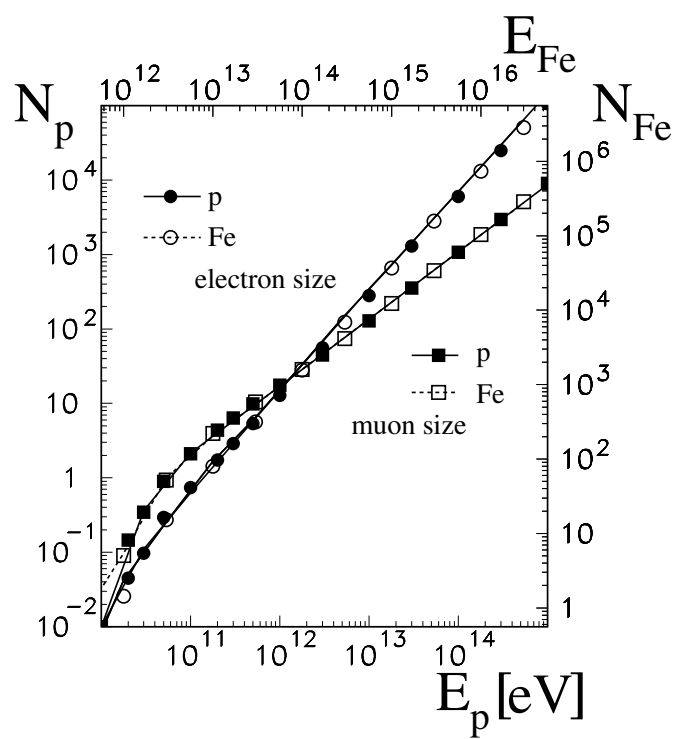

Figure 1: Average electron and muon numbers in CORSIKA showers initiated by protons (solid circles - scales on the left and bottom) and by iron nuclei shifted respectively according to the superposition model (empty circles - scales on the right and on the top). Lines show the results obtained by our "fast small shower generator": solid for protons and dashed for iron initiated showers.

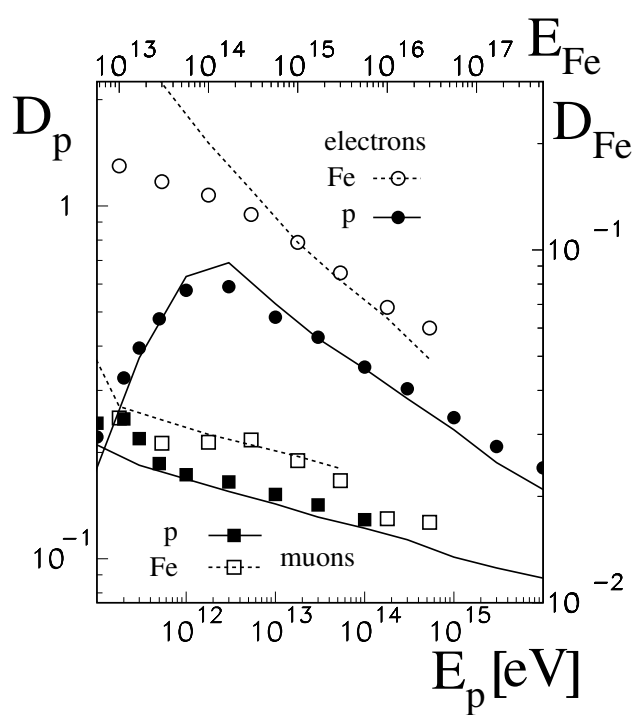

Figure 2: Dispersion of the logarithm of electron and muon numbers in CORSIKA showers initiated by protons and by iron nuclei shifted respectively according to the superposition model of the shower development. Symbols, lines and respective scales are like in Figure 1.

Fig. 1 shows the average values obtained from the simulations for primary protons, and for the iron nuclei. First of all, it is worth to notice, that practically in the whole shown energy range from several times $10^{11} \mathrm{eV}$ to $10^{16} \mathrm{eV}$, the dependence is definitely power-law with different indices for muon and electron size. According to the picture of simple superposition nucleus is treated as a set of single nucleons and a shower initiated by, for example, an iron nucleus is the same as 56 proton showers. This assumption is quite natural and to a large extent correct, as we can see for example, in Fig. 1.Both of these observations (of the CORSIKA results) indicate that the superposition assumption is correct, but this is not entirely and exactly true. Another important characteristic of EAS, which is indispensable for carrying out correct calculations of fluxes of particles in small showers, is the size of the fluctuations of the shower size at small energies of particles which are initiating them. Fig. 2 shows the dispersion of the logarithm of the electron (a) and muon (b) size distributions as a function of the energies of the protons and iron nuclei initiating showers. Energy scales for iron showers (top) correspond to the same energy per nucleon as the respective scales for proton induced showers (bottom). Ordinate for iron showers (right) is scaled down by $\sqrt{56}$. In the picture of simple superposition, the dispersion of 56 independent proton showers would correspond to the expected dispersion for iron induced showers. As can be seen, the points from the simulation calculations with the CORSIKA program for proton initiated showers do not overlap with the correspondingly shifted values for iron showers. The lines in Fig. 2 represent our proposed solution, the results of our 'fast small shower generator'.

It has been known for many years that the transverse distributions of particles in extensive air 

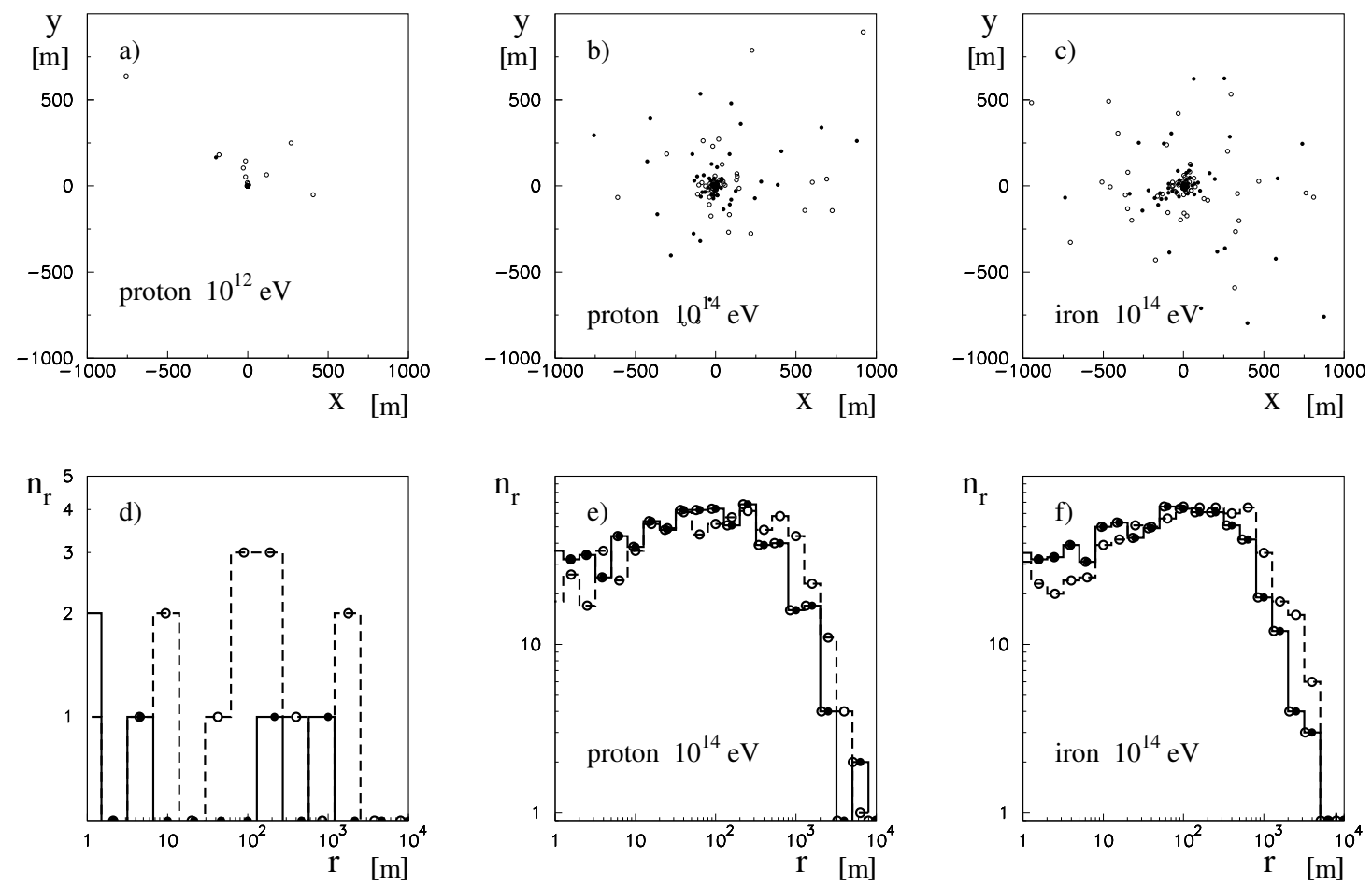

Figure 3: Examples of showers generated by our small shower generator. The $(x \times y)$ distribution of electrons (filled circles) and mouns (empty circles) for proton shower of energies $10^{12} \mathrm{eV}$ (a), and $10^{14} \mathrm{eV} \mathrm{b}$ ) and iron shower with energy of $10^{14} \mathrm{eV} \mathrm{c}$ ) (for b) and c) only 100 particles are plotted). Bottom histograms d), e) and f) show radial distributions of particles in these showers, respectively.

showers are well described by a simple formula proposed by Greisen [18]. Its validity was confirmed by theoretical considerations and numerical calculations with respect to electromagnetic cascades by Kamata and Nishimura [19], hence its commonly accepted name: Nishimura-Kamata-Greisen (NKG) function

We have determined average number of particles in the shower $\langle N\rangle$, logarithmic dispersion of the actual size, and age parameter and "Molière unit" of NKG function, separately for soft (electron) and hard (muon) components of CORSIKA showers as a function of primary particle energy, its mass, and incoming angle.

Next, we have to verify a simple superposition model which allows to eliminate from our descriptions the mass of the primary particle as an independent variable. As we have shown above, this model works well for average shower sizes. We have noticed a significant discrepancy comparing the dispersion (logarithmic) of the total number of particles for protons and iron nuclei. The observed dispersion for iron is greater than expectation obtained by scaling by the square root of the mass number $\sqrt{56}$ spread of the size of the iron shower. This indicates the existence of a correlation between the 'subshower components'.

The simplest introduction of correlations among $A$ independent nucleon (proton) subshowers with the same energy per nucleon they are supposed to mimic is to assume that some of them have identical actual size. This decreases the number of independent compounds, thus increasing the 
dispersion as we wish to.

It is known that the main source of shower size fluctuations is the height of the first interaction, which it is related to the cross section of the interaction, it is reasonable and theoretically justified to assume that the average number of identical sub-shower components is proportional to the number of wounded nucleons in the interaction of the cosmic ray nucleus with the atomic nucleus of the atmosphere. Of course, we are only talking about the wounded nucleon of the beam, cosmic ray nucleus $A$. The actual integer number of identical subshowers in each nucleus induced shower fluctuates according to a binomial distribution.

The results of the modified superposition model are shown in Fig. 2 for electrons and muons. As can be seen, in the region where the shower sizes are sufficiently large, we have obtained a good enough agreement with CORSIKA simulations. As we have said, for very small showers the integer number of particles in single showers and the combination of the physical spread with the effect from the Poisson distribution have a dominant role.

It allows to compare predictions not only with the CORSIKA showers which it is based on, but with experimental results, e.g., the shower particle density spectrum. It has been measured since at least the middle of the last century. The form of the spectrum found agreed with a simple power law formula $\rho^{-\gamma}$, for example the one measured by Cocconi, Loverdo and Tongiorgi in 1946 $(\gamma=1.47)[20,21]$, Broadbent et al. in 1950 $(\gamma=1.425)$ [22], Norman in $1956(\gamma=1.39)$ [23], or Greisen in $1960(\gamma=1.3)$ [18].

With our fast small shower generator one can carry out the multidimensional Monte Carlo integration to obtain the shower particle density observed with a single small detector. The result of the integrationis shown in Fig. 4 in comparison with measured results listed above. As we can

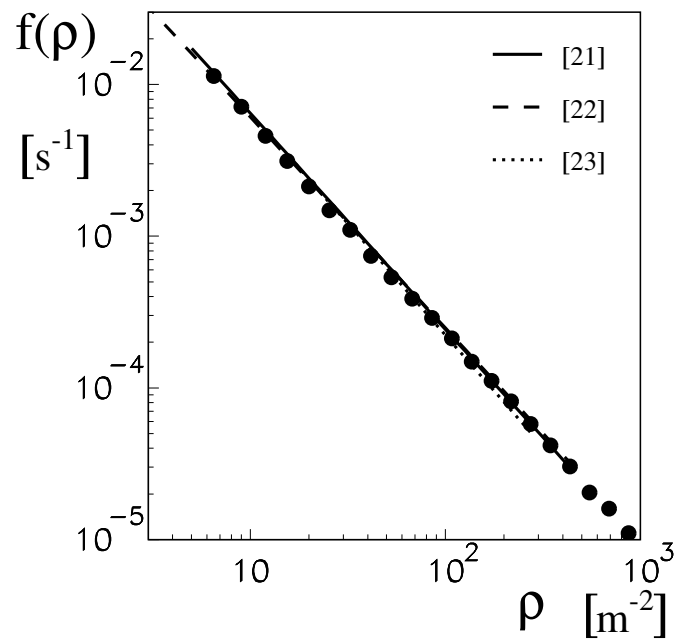

Figure 4: Shower particle density spectra obtained using our small shower generator (circles) compared with measurements [20, 22, 23]. see, the agreement is very good.

All this with the modified superposition approach is already sufficient to formulate a simple algorithm generating small EAS that will resemble the EAS generated by the CORSIKA program. Examples of showers generated in this way are shown in Fig. 3.

If we measure the number of particles observed on a $1 \mathrm{~m}^{2}$ detector, each observed value corresponds to a different distribution of the primary particle energy. The results of the calculation are shown in Fig. 5. These results lead to some important conclusions: for example, rate of registration of single muons is several times higher than rate of registration of single electrons, and opposite, the cases of simultaneous registration of more than one muon are much rarer than those of more than one electron and the primary energy required is much higher. 


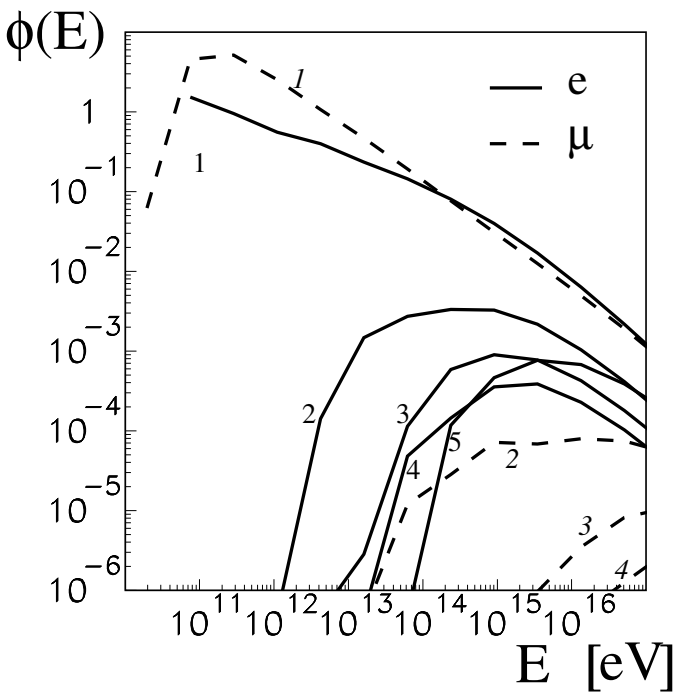

Figure 5: Primary particle energy spectra leading to the observation in a $1 \mathrm{~m}^{2}$ detector of 1,2 , 3,4 and 5 particles: electrons (solid lines) and muons (dashed lines).

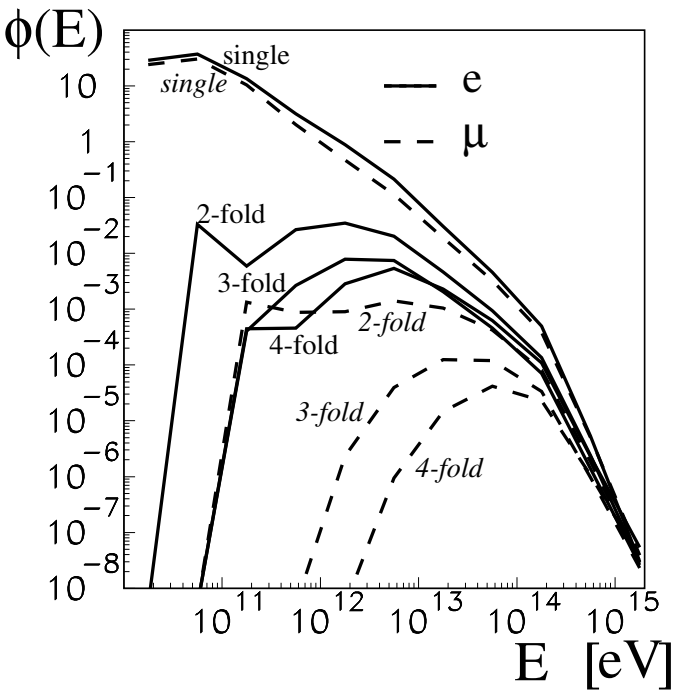

Figure 6: Distributions of primary energies which are responsible for events on $1,2,3$, and $41 \mathrm{~m} \times 1 \mathrm{~m}$ detectors fired with electrons (solid lines) and by muons (dashed lines).

As it was mentioned, the main application of the small shower generator is to assist in the interpretation of data from small shower arrays, either for educational purposes or for use in integrated networks of local stations. For example, let us assume that such stations would consist of four identical detectors located not far from each other. Small shower generator will help to answer the question with what energy of the primary particle, or more precisely with what energy distribution one should associate a given type of coincidence.

Examples of such results are presented in Fig. 6.

\section{Conclusions}

We developed the "small shower generator", which can be used as a semianalytical method for the calculation of secondary particle flux at the sea level and to mimic the exact shower generation provided by CORSIKA and other Monte-Carlo programs which fully simulate a shower passing through the Earth's atmosphere. More details are given in [24].

Using our small shower generator, it is possible to perform fast integrations of secondary particle fluxes at sea level and predict the registrations made by small school local EAS arrays as well as single detectors. These predictions, when confronted with the measured values, allow a deeper analysis of the local measurements and the properties of the detectors themselves. The small shower generator also allows the count rate in small EAS arrays to be estimated and the detector size and number of detectors to be optimalised in this respect.

\section{References}

[1] Linsley, J., Mini and Super Mini Arrays for the Study of Highest Energy Cosmic Rays, Proceedings, $19^{\text {th }}$ International Cosmic Ray Conference (ICRC1985) La Jolla, US OG9.4-9 434. 
[2] van Dam, K. et al., The HiSPARC Experiment, astro-ph/1908.01622.

[3] Wilkes, R.J. et al. WALTA school-network cosmic ray detectors, IEEE Transactions on Nuclear Science, $\mathbf{5 1 , 1 3 8 5 .}$

[4] Brouwer, W. et al. The ALTA cosmic ray experiment electronics system Nuclear Instruments and Methods in Physics Research Section A: Accelerators, Spectrometers, Detectors and Associated Equipment" 539 595;

[5] Smolek, K., Nyklíček, M., Kovačíková, P., Cosmic Rays \& Experiment CZELTA, AIP Conference Proceedings 958197.

[6] Brobeck, E., Measurement of ultra-high energy cosmic rays with CHICOS, Dissertation (Ph.D.), California Institute of Technology.

[7] Franke, R. et al. CosMO - A Cosmic Muon Observer Experiment for Students Proceedings, $33^{\text {rd }}$ ICRC2013, Rio de Janeiro, Brazil, 1084

[8] Gawin, J. et al. The Roland Maze Project, Acta Physics Polonica B 33349.

[9] Feder, J. et al.The Roland Maze Project - Cosmic Ray Registration At Schools International Journal of Modern Physics A 206881.

[10] Auger, P., Maze, R., Grivet-Mayer, T. Heavy electrons in cosmic ray showers originating in the atmosphere Compt. Rend. Acad. Sci. B206 1721.

[11] Auger, P. and Maze, T. Heavy electrons in cosmic ray showers originating in the atmosphere Compt. Rend. Acad. Sci. B207 288.

[12] N.M. Gerasimova, N.M. and Zatsepin, G.T., Splitting of cosmic ray nuclei by solar photons, Soviet Phys., JETP 11899.

[13] Medina-Tanco, G.A. and Watson, A.A., The photodisintegration of cosmic ray nuclei by solar photons: the Gerasimova-Zatsepin effect revisited Astroparticle Physics 10157.

[14] Heck, D., CORSIKA: a Monte Carlo code to simulate extensive air showers. report KZKA6019, Karlsruhe.

[15] Heck, D. and Pierog, T., Extensive Air Shower Simulationwith CORSIKA: A User's Guide (Version 7.7402), Karlsruher Institute fur Technologie Report from December 18, 2020.

[16] Antoni, T. et al. The cosmic-ray experiment KASCADE, Nuclear Instruments and Methods in Physics Research Section A: Accelerators, Spectrometers, Detectors and Associated Equipment 513, 490.

[17] Klages, H.O. et al. The Extensive Air Shower Experiment Kascade - First Results Proceedings, $25^{\text {th }}$ International Cosmic Ray Conference (ICRC1997) Durban, South Africa, 1997.

[18] Greisen, K., Cosmic Ray Showers, Annual Review of Nuclear Science 1063.

[19] Kamata, K. and Nishimura, J., The Lateral and the Angular Structure Functions of Electron Showers Progress of Theoretical Physics Supplement 6, 93.

[20] Cocconi, G. and Loverdo, A. and Tongiorgi, V., The Density Spectrum of the Extensive Cosmic-Ray Showers of the Air, Phys. Rev. 70, 841.

[21] Cocconi, G. and Loverdo, A. and Tongiorgi, V., Experimental and Theoretical Evaluation of the Density Spectrum of Extensive Cosmic-Ray Showers Phys. Rev. 70, 846.

[22] Broadbent, D., Kellermann E.W., and Hakeem, M.A., The Density Spectrum and Structure of Extensive Cosmic-ray Air Showers at Sea Level Proceedings of the Physical Society A 63864.

[23] Norman, R.J. The Energy Spectrum of Extensive Air Showers Proceedings of the Physical Society A69, 11804.

[24] Wibig, T., Small shower CORSIKA simulations Chinese Physics C 45085001. 